\title{
A New Approach to High Sensitivity Liquid Chromatography-Mass Spectrometry of Peptides using Nanoflow Solvent Assisted Inlet Ionization
}

\author{
Beixi Wang, Ellen D. Inutan, Sarah Trimpin \\ Department of Chemistry, Wayne State University, Detroit, MI 48202, USA
}

\begin{abstract}
Liquid chromatography (LC) solvent assisted inlet ionization (SAll) mass spectrometry (MS) was previously reported to give good chromatographic resolution and MS detection injecting $66 \mathrm{ng}$ of a BSA tryptic digest. In analogy to nano-electrospray ionization ( $\mathrm{nESI}$ ), we extend SAII LC/MS to nano-SAll (nSAll) operating at $\mathrm{nL} \mathrm{min}^{-1}$ flow rates and demonstrate good quality ion chromatograms and mass spectra from injection of as little as $0.7 \mathrm{ng}$ of BSA digest onto a capillary LC column. Data dependent fragmentation is demonstrated for injection of $7 \mathrm{ng}$ of a BSA digest. This method has advantages over $\mathrm{nESI}$ in ease of use and low cost as it requires no voltage and is operational without the necessity of connectors or fragile $\mathrm{nESI}$ emitters, although similar constricted tips can be helpful in nSAll to stabilize the signal at low nanoliter flow. At a flow rate of $0.8 \mu \mathrm{Lmin}^{-1}$, the only requirement for nSAll is that the exit-end of the capillary LC column be adjusted near the aperture of the heated inlet of the mass spectrometer.
\end{abstract}

Key words: Inlet ionization, Nano-solvent assisted inlet ionization (nSAll), Liquid chromatography (LC), Mass spectrometry (MS), data dependent MS/MS, BSA tryptic digest, Nano-electrospray ionization (nESI)

\section{Introduction}

$\mathrm{M}$ odern liquid chromatography/mass spectrometry (LC/MS) almost exclusively uses electrospray ionization (ESI) except for low polarity compounds which use atmospheric pressure chemical ionization (APCI) [1-3]. For analyses where sample amount is limited, ESI LC/MS is performed at nanoliter $(\mathrm{nL}) \mathrm{min}^{-1}$ mobile phase flow rates [4-7]. This approach, called nano-ESI (nESI) [8], is especially important for peptide and protein analyses. Because ESI is a concentration sensitive method, lower flow rates produce similar signal to higher flow but with consumption of less material [9]. However, the low flow

Electronic supplementary material The online version of this article (doi:10.1007/s13361-011-0320-8) contains supplementary material, which is available to authorized users.

Correspondence to: Sarah Trimpin; e-mail: strimpin@chem.wayne.edu condition also enhances ionization, presumably by producing smaller solvent droplets with increased charge, thus reducing ion suppression and ionizing a wider range of compounds. Because of the importance of nESI, ion sources are commercially available. Recently, Smith and coworkers $[10,11]$ reported on nESI at subambient pressure using ion funnel technology. However, nESI has a number of shortcomings. The low solvent flow greatly increases the time for a complete LC run relative to higher flow rates, and achieving a stable ion current is notoriously difficult. Special spray emitters are available to help address this issue [12], but the emitter tips are fragile and add considerable cost to nESI. The sharp tips also reduce the voltage range over which a stable ESI spray is observed: too high voltage produces a corona discharge. Technical issues generally limit the use of nESI to problems that need its additional capabilities.

Recently, inlet ionization methods have been introduced that have the potential to compete with both matrix assisted laser desorption/ionization (MALDI) and ESI. Methods that utilize a 
solid phase matrix similar to those used in MALDI are laserspray ionization inlet (LSII) [13-19] and matrix assisted inlet ionization (MAII) [20]. Solvent assisted inlet ionization (SAII) is the equivalent of ESI in that similar mass spectra are produced from solvent/analyte solutions introduced into the mass spectrometer inlet and ionized [21]. Just as in ESI, SAII can be used for ionization in LC/MS [22]. It was shown that introducing the $\mathrm{LC}$ mobile from a $1 \mathrm{~mm}$ i.d. LC column directly into a heated inlet transfer tube of the mass spectrometer produced a high quality ion chromatogram and corresponding mass spectra with injection of ca. $70 \mathrm{ng}$ of bovine serum albumin (BSA) tryptic digest. Here, we extend the applicability of inlet ionization to nanoliter flow rates by positioning the LC effluent just outside of the mass spectrometer inlet aperture and demonstrate similar results from injection of just $0.7 \mathrm{ng}$ of BSA digest.

\section{Experimental}

\section{Materials}

Trypsin-digested BSA MS standard (CAM-modified) was purchased from New England BioLabs Inc. (Ipswich, MA, USA). Angiotensin I was purchased from American Peptide Company, Inc. (Sunnyvale, CA, USA). Formic acid (FA) and acetonitrile (ACN) were obtained from Fisher Scientific Inc. (Pittsburgh, PA, USA). HPLC grade water was obtained from EMD chemicals (Gibbstown, NJ, USA).

\section{SAII-MS}

One end of a $40 \mathrm{~cm}$ length of $25 \mu \mathrm{m}$ i.d. fused silica tubing (Polymicro Technologies, Phoenix, AZ, USA) was connected to a syringe and the exit end taped on an $\mathrm{x}, \mathrm{y}, \mathrm{z}$-stage to control the alignment and distance of the exit end relative to the inlet aperture of the heated atmospheric pressure (AP) to vacuum inlet tube of the mass spectrometer. A study of the optimum distance of the fused silica exit from the inlet orifice was performed by pumping a $1: 1 \mathrm{ACN}$ :water $0.1 \%$ FA 1 pmol $\mu \mathrm{L}^{-1}$ angiotensin I solution through the fused silica tube at a flow rate of $1.2 \mu \mathrm{L} \mathrm{min}^{-1}$. The $\mathrm{x}, \mathrm{y}, \mathrm{z}$-stage was used to adjust the exit end of the fused silica from $0.4 \mathrm{~mm}$ on the AP side to $0.2 \mathrm{~mm}$ on the vacuum side of the mass spectrometer inlet aperture.

\section{NanoSAII and nanoESI LC-MS and MS/MS}

A Waters Corporation NanoAcquity liquid chromatograph was used with a Waters $100 \mu \mathrm{m} \times 100 \mathrm{~mm}$ BEH130 C18 column with $1.7 \mu \mathrm{m}$ particles. Water and $\mathrm{ACN}$ both containing $0.1 \% \mathrm{FA}$ was used as the mobile phases in all studies. A 35 min gradient of 1 to $85 \% \mathrm{ACN}$ was used at mobile phase flow rates varying from 0.4 to $0.8 \mu \mathrm{L} \mathrm{min}{ }^{-1}$, and a 12 min gradient at a flow rate of $1.2 \mu \mathrm{L}$ $\min ^{-1}$. For $0.4 \mu \mathrm{L} \mathrm{min}^{-1}$ flow rate, a $6.35 \mathrm{~cm}$ long $360 \mu \mathrm{m}$ o.d. $\times$ $20 \mu \mathrm{m}$ i.d. (with $10 \mu \mathrm{m}$ i.d. at the tip) pre-cut PicoTip emitter (Waters) was employed as an extension to the end of LC capillary column and mounted on the $\mathrm{x}, \mathrm{y}, \mathrm{z}$-stage to be positioned about $0.1-0.2 \mathrm{~mm}$ outside of the mass spectrometer inlet for optimum results. At flow rates of $0.8 \mu \mathrm{L} \mathrm{min}^{-1}$ and higher, the exit-end of the LC capillary column $(100 \mu \mathrm{m}$ i.d. $\times 360 \mu \mathrm{m}$ o.d. $)$ was used without need of a special emitter or any tubing connections. A Thermo LTQ-Velos mass spectrometer with the inlet tube heated to $300{ }^{\circ} \mathrm{C}$ was used for LC-nSAII-MS and MS/MS. Data dependent MS/MS was obtained using collision induced dissociation (CID) with $35 \mathrm{~V}$ collision energy. A three point boxcar smoothing was used for the LC chromatogram display. A Waters SYNAPT G2 mass spectrometer [17, 18, 20, 22] was used for LC-nESI-MS for relative comparison. The skimmer cone was heated to $150{ }^{\circ} \mathrm{C}$. The voltage applied on the nESI capillary is $3.06 \mathrm{kV}$. BSA tryptic digest solution, $1 \mu \mathrm{L}$ of a $100 \mathrm{fmol} \mu \mathrm{L}^{-1}$, was analyzed using the same LC gradient at a mobile phase flow of $0.8 \mu \mathrm{L} \mathrm{min}{ }^{-1}$ and $0.4 \mu \mathrm{L} \mathrm{m^{-1 }}$.

\section{Results and Discussion}

SAII was recently interfaced with LC/MS at a mobile phase flow rate of $55 \mu \mathrm{L} \mathrm{min}^{-1}$ with chromatographic resolution comparable to ESI LC/MS and produces good signal-to-noise with injection of 1 pmol of a BSA digest [22]. This work was accomplished on a LTQ-Velos, which has a heated inlet tube
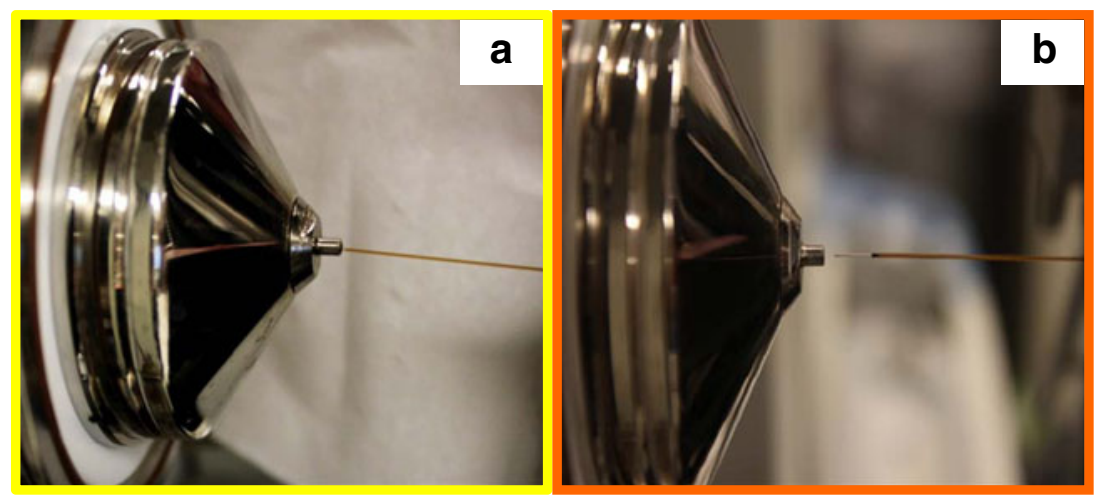

Figure 1. Pictures showing the setup for LC-nSAll. Side view of MS inlet in which the tip of the fused silica capillary tube of the LC column is placed about $0.1 \mathrm{~mm}$ out of the orifice inlet entrance of the mass spectrometer (a) directly or (b) by use of a "PicoTip" attached to the end of the fused silica capillary tube. Front view of the LC and mass spectrometer setup is displayed in Figure S3 


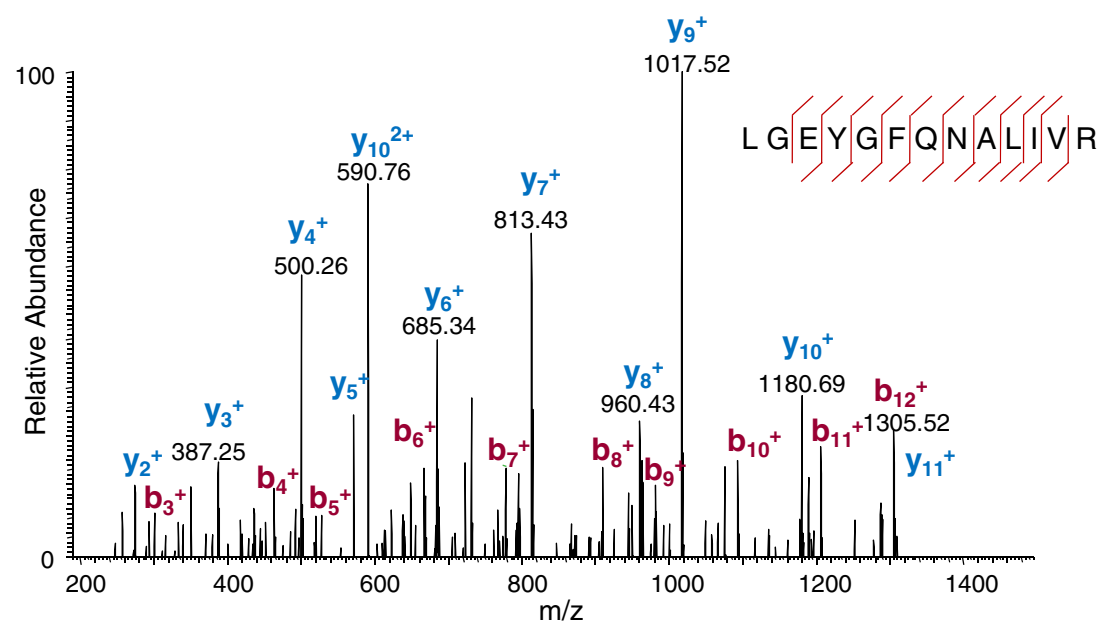

Figure 2. LC-nSAll-MS/MS mass spectrum of $100 \mathrm{fmol} \mu \mathrm{L}^{-1} \mathrm{BSA}$ tryptic digest with $1 \mu \mathrm{L}$ injection at a flow rate of $800 \mathrm{~nL} \mathrm{~min}^{-1}$ using the setup shown in Figure 1(a). The sequence shows the Mascot coverage providing a MASCOT score of 103

linking AP with the first vacuum region of the mass spectrometer. The higher flow rates used in the previous study requires that the exit-end of the fused silica capillary be stripped of its polyimide coating and inserted into the heated inlet tube of the mass spectrometer to a 'sweet spot' for ion production. Here, we demonstrate, in analogy to nESI, that nanoliter mobile phase flow rates are also compatible with SAII. The initial 'tuning' for the low flow rate was achieved by delivering an angiotensin I solution using an infusion pump to the exit end of the fused silica tube $(25 \mu \mathrm{m}$ i.d.) so that it could be adjusted (Figure S1) for the best stability and abundance of the signal from the triply charged angiotensin I ion $(\mathrm{m} / \mathrm{z} 433)$. For flow rates of a few microliters and lower, the best position of the fused silica capillary exit-end was found to just at the inlet entrance aperture, and no longer requiring the removal of the coating. A stable signal with good sensitivity was achieved with the mass spectrometer inlet tube temperature set to $300{ }^{\circ} \mathrm{C}$. The flow of air into the vacuum region of the heated mass spectrometer entrance inlet is sufficient to nebulize the solution at the tip of the fused silica, sweeping the ensuing mist of solvent droplets into the inlet where ions are generated with the assistance of heat and vacuum. Experiments and mechanistic discussions are included in Supplemental Data (Figure S2).

Without the need to place the fused silica inside the heated mass spectrometer inlet, it is possible to use the exit of the capillary LC column and eliminate all connections, unlike in our previous LC-SAII study [22]. By attaching the exit of the LC column to an $\mathrm{x}, \mathrm{y}, \mathrm{z}$-stage with tape, the end of the LC column capillary tubing is visually adjusted near the entrance of the mass spectrometer inlet for optimum ion current stability (Figure 1a and Figure S3). This procedure can be accomplished in a couple of minutes. This arrangement provides stable ion current at flow rates from $0.8 \mu \mathrm{L} \mathrm{min}{ }^{-1}$ (Figure $\mathrm{S} 4 \mathrm{~A}$ ) to at least $1.2 \mu \mathrm{L} \mathrm{\textrm {min } ^ { - 1 }}$ (Figure S4B). However, the signal is not sufficiently stable with this arrangement at $0.4 \mu \mathrm{L} \mathrm{min}{ }^{-1}$ for good quality LC/MS. Observationally, it appears that the instability is the result of larger droplets exiting the fused silica tubing rather than a fine spray. A stable signal is achieved by attaching the LC column to a Waters PicoTip emitter (Figure 1b), as is done in nESI. Because SAII operates without the requirement for a voltage and at high nanoliter flow rates without the necessity of a fragile emitter, it is exceptionally easy to implement.

Using the simple set-up (Figure 1a and Figure S3) requiring no emitter connected to the LC column and a mobile phase flow rate of $0.8 \mu \mathrm{L} \mathrm{min}^{-1}$, injection of $100 \mathrm{fmol}(7 \mathrm{ng}$ ) of BSA digest produces roughly equivalent results to those reported [22] for ca. $70 \mathrm{ng}$ injected at $55 \mu 1 \mathrm{~min}^{-1}$. The base peak chromatogram for the 100 fmol injection is shown in Figure S5A. The mass spectrum from the peak eluting at 17.8 min shows doubly and singly charged analyte ions (Figure S5B). In order to have a relative comparison, $\mathrm{nESI}$ LC/MS was acquired using the commercial nESI source on the SYNAPT G2 mass spectrometer at a mobile phase flow rate of 0.8 and $0.4 \mu \mathrm{L} \mathrm{min}{ }^{-1}$ using the same gradient and injecting $100 \mathrm{fmol}$ of the same BSA digest used in the SAII study (Figures S4 and S6).

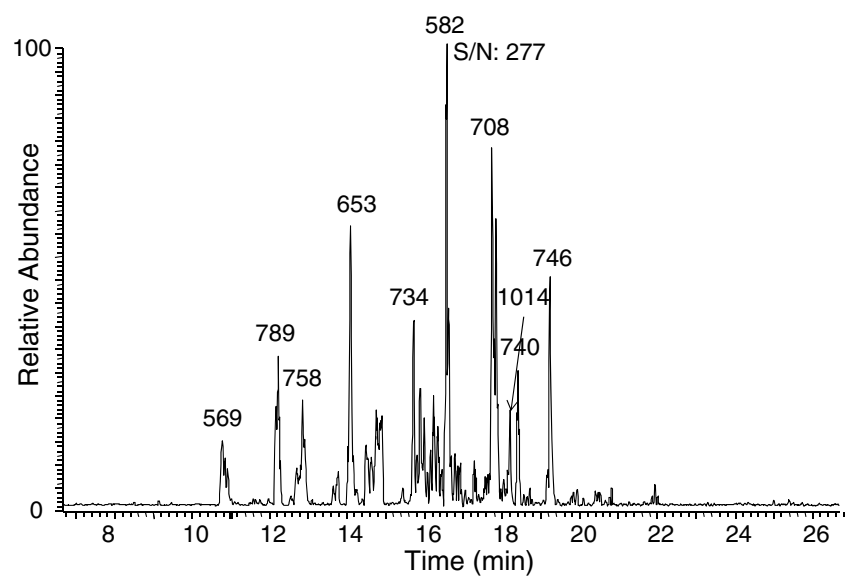

Figure 3. nSAll-LC/MS base beak chromatograms of $10 \mathrm{fmol}$ $\mu \mathrm{L}^{-1}$ BSA tryptic digest with $1 \mu \mathrm{L}$ injection at a flow rate of

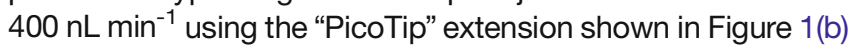


Additionally, data dependent fragmentation for the $100 \mathrm{fmol}$ SAII injection on LTQ Velos also produces excellent LC/MS/ MS fragmentation as is demonstrated by the high sequence coverage using MASCOT for the BSA peptide fragment at $\mathrm{m} / \mathrm{z}$ 740 having the sequence LGEYGFQNALIVR (Figure 2). MASCOT identified the protein as BSA from this single MS/ MS spectrum with a score of 103 . The first nSAII LC/MS and MS/MS results, without the necessity of special connections, tips, or voltage, produce roughly equivalent results to $\mathrm{nESI}$ and in much less set-up time for the experiment.

Injection of just $10 \mathrm{fmol}(0.7 \mathrm{ng})$ of BSA digest using SAII at $0.8 \mu \mathrm{L} \mathrm{min}^{-1}$ produces the base peak chromatogram in which only the most abundant ions are observed. However, connecting the exit of the LC column to a $20 \mu \mathrm{m}$ i.d. PicoTip with $10 \mu \mathrm{m}$ i. d. on the tip allows a stable signal to be obtained at a flow rate of $0.4 \mu \mathrm{L} \mathrm{min}{ }^{-1}$. Under these conditions, injection of $0.7 \mathrm{ng}$ of BSA digest produced the base peak chromatogram shown in Figure 3. The signal-to-noise was calculated by the data system for the peak eluting at $m / z 582$ is 277 .

\section{Conclusion}

Inlet ionization is a new method for producing mass spectra equivalent in charge state to those produced by ESI but from solid (LSI or MAII) or solution (SAII) states without the need for a laser or voltage for ionization. The sensitivity of inlet ionization is demonstrated by the production of good quality base peak chromatograms and clean mass spectra with high signal-to-noise with injection of as little as $0.7 \mathrm{ng}$ of a BSA digest using nSAII LC/MS. Data-dependent fragmentation was shown for injection of just $7 \mathrm{ng}$ of BSA digest using a mobile

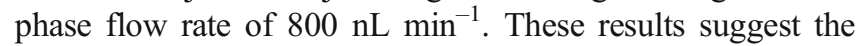
potential of SAII for proteomics and most likely other areas where sample amounts are limited. Detailed mechanistic discussion relative to inlet ionization is addressed in a forthcoming paper [23].

\section{Acknowledgement}

The authors are grateful for competitive discussion with Professor Charles McEwen, University of the Sciences, and the funding from NSF CAREER 0955975, ASMS (Waters Company) Research Award, and DuPont Young Professor Award (to S.T.) and Schaap Fellowship (to E.D.I.).

\section{References}

1. Wachs, T., Conboy, J., Henion, J.: Liquid chromatography-mass spectrometry and related techniques via atmospheric pressure ionization. $J$. Chromatogr. Sci. 29, 357-366 (1991)

2. Niessen, W., Tinke, A.: Liquid chromatography-mass spectrometry, general principles and instrumentation. J. Chromatogr. A 703, 37-57 (1995)
3. Mehlis, B., Kertscher, U.: Liquid chromatography/mass spectrometry of peptides of biological samples. Anal. Chim. Acta 352, 71-83 (1997)

4. Srinivas, P., Krishna, P., Sadanandam, M.: Nano liquid chromatography in pharmaceutical analysis-a review. Int. J. Ph. Sci. 2, 728-737 (2010)

5. Shenm, Y., Tolic, N., Masselon, C., Pasa-Tolic, L., Camp, D.G., Hixson, K.K., Zhao, R., Anderson, G.A., Smith, R.D.: Ultrasensitive proteomics using high-efficiency on-line micro-SPE-nanoLC-nanoESI MS and MS/MS. Anal. Chem. 76, 144-154 (2004)

6. Shen, Y., Zhao, R., Berger, S.J., Anderson, G.A., Rodriguez, N., Smith, R.D.: High-efficiency nanoscale liquid chromatography coupled on-line with mass spectrometry using nanoelectrospray ionization for proteomics. Anal. Chem. 74, 4235-4249 (2002)

7. Smith, R.D., Shen, Y., Tang, K.: Ultrasensitive and quantitative analyses from combined separations-mass spectrometry for the characterization of proteomes. Acc. Chem. Res. 37, 269-278 (2004)

8. Mann, W.M.: Analytical properties of the nanoelectrospray ion source. Anal. Chem. 68, 1-8 (1996)

9. Smith, R.D.: Future directions for electrospray ionization for biological analysis using mass spectrometry. Biotechniques 41, 147-148 (2006)

10. Tang, K., Page, J.S., Marginean, I., Kelly, R.T., Smith, R.D.: Improving liquid chromatography-mass spectrometry sensitivity using a subambient pressure ionization with nanoelectrospray (SPIN) interface. J. Am. Soc. Mass Spectrom. 22, 1318-1325 (2011)

11. Page, J.S., Tang, K., Kelly, R.T., Smith, R.D.: Subambient pressure ionization with nanoelectrospray source and interface for improved sensitivity in mass spectrometry. Anal. Chem. 80, 1800-1805 (2008)

12. Gibson, G.T.T., Mugo, S.M., Oleschuk, R.D.: Nanoelectrospray emitters trends and perspective. Mass Spectrom. Rev. 28, 918-936 (2009)

13. Trimpin, S., Herath, T.N., Inutan, E.D., Cernat, S.A., Wager-Miller, J., Mackie, K., Walker, J.M.: Field-free transmission geometry atmospheric pressure matrix-assisted laser desorption/ionization for rapid analysis of unadulterated tissue samples. Rapid Commun. Mass Spectrom. 23, 30233027 (2009)

14. Trimpin, S., Inutan, E.D., Herath, T.N., McEwen, C.N.: Laserspray ionization-A new AP-MALDI method for producing highly charged gas-phase ions of peptides and proteins Directly from solid solutions. Mol. Cell. Proteom. 9, 362-367 (2010)

15. Trimpin, S., Inutan, E.D., Herath, T.N., McEwen, C.N.: A Matrixassisted laser desorption/ionization mass spectrometry method for selectively producing either singly or multiply charged molecular ions. Anal. Chem. 82, 11-15 (2010)

16. Inutan, E.D., Richards, A.L., Wager-Miller, J., Mackie, K., McEwen, C. N., Trimpin, S.: Laserspray ionization - a new method for protein analysis directly from tissue at atmospheric pressure and with ultra-high mass resolution and electron transfer dissociation sequencing. Mol. Cell. Proteom. 10, 1-8 (2011)

17. Inutan, E.D., Trimpin, S.: Laserspray ionization (LSI) ion mobility spectrometry (IMS) mass spectrometry (MS). J. Am. Soc. Mass Spectrom. 21, 1260-1264 (2010)

18. Inutan, E.D., Trimpin, S.: Laserspray ionization - ion mobility spectrometry - mass spectrometry: Baseline separation of isomeric amyloids without the use of solvents desorbed and ionized directly from a surface. J. Proteome Res. 9, 6077-6081 (2010)

19. Richards, A.L., Lietz, C.B., Trimpin, S.: Imaging Mass Spectrometry in Transmission Geometry. Rapid Commun. Mass Spectrom. 25, 815-829 (2011)

20. McEwen, C.N., Pagnotti, V., Inutan, E.D., Trimpin, S.: A new paradigm in ionization: multiply charged ion formation from a solid matrix without a laser or voltage. Anal. Chem. 82, 9164-9168 (2010)

21. Pagnotti, V.S., Chubatyi, N.D., McEwen, C.N.: Solvent assisted inlet ionization: an ultrasensitive new liquid introduction ionization method for mass spectrometry. Anal. Chem. 83, 3981-3985 (2011)

22. Pagnotti, V.S., Inutan, E.D., Marshall, D.D., McEwen, C.N., Trimpin, S.: Inlet ionization: a new highly sensitive approach for liquid chromatography-mass spectrometry of small and large molecules. Anal. Chem. 83, 7591-7594 (2011)

23. Trimpin, S., Wang, B., Inutan, E.D., Li, J., Lietz, C.B., Pagnotti, V.S., Harron, A.F., Sardelis, D., McEwen, C.N.: A unified mechanism for ionization of nonvolatile compounds in mass spectrometry: Lessons from MALDI and LSI, J. Am. Soc. Mass Spectrom. (submitted). 